\title{
Changing Paradigms in Breast Cancer Therapeutics: An Extended Abstract
}

\author{
Jo Anne Zujewski
}

National Cancer Institute, Bethesda, Md., USA

Breast cancer is the leading cause of cancer in women worldwide, with an incidence of 1,671,000 and a mortality of 522,000 according to GLOBOCAN 2012 [1]. Over the past several decades, breast cancer has increased in incidence, but in regions of the world with access to cancer care, mortality has decreased. During the same time, our understanding of the pathophysiology of breast cancer has changed dramatically.

In the late 1800s, the standard treatment of breast cancer was radical mastectomy. This approach was based on an understanding of cancer as an anatomical disease. In short, the thinking at the time was that cancer spread in an orderly fashion from the breast to the regional lymph nodes and from there to distant sites. This understanding led to the development of Halsted's radical mastectomy - more extensive surgeries were thought to remove the cancerous cells before the cells had time to spread. However, not all surgeons at the time of Halsted were able to replicate Halsted's results, and it was noted that even patients with small tumors and radical surgery would sometimes experience a recurrence. It took over a century to perform randomized clinical trials demonstrating that breast cancer is a systemic disease and therefore extensive surgical therapy did not improve survival. In the 1970s, randomized clinical trials were initiated to resolve controversy over the surgical management of breast cancer. After 20 years of follow-up, no significant difference in overall survival was found between women who underwent mastectomy and those who underwent lumpectomy with or without postoperative breast irradiation $[2,3]$.

\section{KARGER}

E-Mail karger@karger.com www.karger.com/mpp
(C) 2015 S. Karger AG, Basel

This is an Open Access article licensed under the terms of the Creative Commons Attribution-NonCommercial 3.0 Unported license (CC BY-NC) (www.karger.com/OA-license), applicable to the online version of the article only. Distribution permitted for non-commercial purposes only.
These trials showed that invasive breast cancer has the ability to spread systemically very early, even before a cancer can be detected on mammographic imaging. Subsequently, clinical trial findings have shown that the use of postmastectomy radiation therapy improves survival in a subset of patients. Therefore, at least in some patients, better local (anatomic) control in addition to systemic therapy may result in an improvement in survival.

In 2000, the US National Institutes of Health conducted a consensus conference. Key recommendations from this conference included that women with estrogen and/ or progesterone receptor-positive breast cancer should receive hormonal therapy (tamoxifen). In addition, most women diagnosed with early-stage breast cancer should receive combination chemotherapy. Although it was clear that not all women required chemotherapy, there was no reliable way to determine which women were able to be treated successfully with local therapy (surgery or surgery and radiation) and hormonal therapy alone, and which women required the addition of chemotherapy.

One of the greatest developments in the 21st century has been the development of a better understanding of the impact of genetic profiles on health and disease. In the clinical laboratory, breast tumors are tested for the hormone receptors (estrogen and progesterone) using immunohistochemistry, and for HER2 (human epidermal growth factor receptor 2) overexpression using either immunohistochemistry or fluorescent in situ hybridization. Advances in genetic or molecular profiling have provided increasing evidence that breast cancer is not a single disease process 
but multiple related cancers with differing molecular aberrations [4]. Based on the gene expression profile in tumor cells, breast cancer can be characterized into at least 5 different molecular subtypes: luminal A, luminal B, HER2enriched, basaloid, and normal. In general, luminal A and $\mathrm{B}$ tumors are hormone receptor-positive, but the clinical phenotyping using just 3 biomarkers does not identify breast tumors into the more detailed molecular subtypes. Each subtype also exhibits marked heterogeneity.

The benefit of adjuvant systemic therapy for early-stage (operable) breast cancer is clearly evident; however, it was noted that some women who would derive benefit from certain adjuvant therapies were not getting them and, conversely, some women were receiving therapies from which they derived little or no benefit. This was of particular concern with the use of chemotherapy, given the toxicity of these agents. In the year 2000, one of the most pressing questions facing oncologists was the identification of hormone receptor-positive patients who could be spared chemotherapy. Although the addition of chemotherapy to hormonal therapy was shown to benefit estrogen receptorpositive, lymph node-negative breast cancer patients in a randomized phase 3 clinical trial [5], it was clear that chemotherapy did not benefit all patients and that the absolute benefit for some individuals was very small. Patients who present estrogen and/or progesterone receptor-positive tumors (approximately two-thirds of breast cancer patients) should receive at least 5 years of hormonal therapy - currently tamoxifen (with or without ovarian function suppression) and/or aromatase inhibitors [6-9].

A molecular profiling assay that can predict the risk of recurrence in patients with hormone receptor-positive node-negative breast cancer has been developed that can help to determine whether adjuvant chemotherapy will be of additional benefit when added to hormonal therapy. This assay, the 21-gene recurrence score, is commercially available as the Oncotype $\mathrm{DX}^{\mathrm{TM}}$ Breast Cancer Assay from Genomic Health (Redwood City, Calif., USA; http:// www.oncotypedx.com). It utilizes a 21-gene panel reverse transcriptase polymerase chain reaction assay comprised of 16 cancer-related genes and 5 internal controls [10].

A prospective trial designed to better refine the value of chemotherapy in women with a low or intermediate recurrence score has been conducted. This trial, the Trial Assessing Individualized Options for Treatment for breast cancer (TAILORx) has completed accrual (NCT00310180; https://clinicaltrials.gov). Results for the low score group should be available in 2015 and for those in the intermediate group in 2017. Currently, a large randomized clinical trial of adjuvant chemotherapy in hor- mone receptor-positive, lymph node-positive early-stage breast cancer patients is ongoing (NCT0127203; https:// clinicaltrials.gov). This trial hypothesizes that it is the biology of the tumor, rather than the anatomic stage, that is the key consideration in determining optimal treatment.

A second major subtype of breast cancer is HER2-positive breast cancer [11]. In 2005, a major advance was made in the treatment of this disease with the publication of the results of a trial of a targeted therapy, trastuzumab, in the treatment of HER2-positive early-stage breast cancer. Trastuzumab is a partially humanized monoclonal antibody directed against HER2. The use of trastuzumab in early-stage breast cancer has turned a disease with a poor prognosis into one with a good prognosis [12-14].

Trastuzumab has also improved the prognosis of women with advanced, metastatic breast cancer [11]. Although the use of trastuzumab has not been curative in metastatic breast cancer patients, it has improved both progression-free and overall survival in these women. Additional advances have been made by the dual blockade of HER2. The HER family members, HER1 and HER2, can dimerize and trigger tyrosine kinase activity, which can result in the activation of downstream pathways involved in cell proliferation, survival and other pathways (including angiogenesis) that are vital to the growth of breast cancers. Pertuzumab is a monoclonal antibody that binds to a different area of HER2 than trastuzumab and prevents dimerization. The randomized phase 3 trial, CLEOPATRA, tested the dual inhibition of HER 2 by pertuzumab and trastuzumab in combination with chemotherapy with docetaxel [15]. The results of this study showed that both progression-free survival and overall survival were improved in the pertuzumab group (pertuzumab plus trastuzumab plus docetaxel) compared to the control group (placebo plus trastuzumab plus docetaxel). A phase 3 trial in women with early-stage breast cancer (APHINITY) has completed accrual (NCT01358877; https://clinicaltrials.gov).

Other strategies for combined targeted therapy in HER2-positive breast cancer include the dual blockade of both the HER2 and estrogen receptor pathways (NCT02003209; https//:clinicaltrials.gov), the inhibition of pathways downstream to HER2 and the use of trastuzumab-drug conjugates (T-DM1, a trastuzumab-emtansine conjugate; NCT00829166; https//:clinicaltrials.gov). A major challenge in this area, as in that of hormonepositive breast cancer, is to identify those patients for whom the dual blockade of HER2 or the HER2 pathway is warranted and those who are adequately treated with trastuzumab.
Zujewski 
In addition to molecular aberrations that occur spontaneously in tumor cells, some individuals inherit a mutated gene that predisposes them to the development of cancer. Examples of these are the breast cancer genes, BRCA1 and BRCA2. These individuals are born with only one functioning BRCA gene, making the likelihood of losing the function of the second gene greater, and thus $50-85 \%$ of women with deleterious mutations in BRCA1 or BRCA2 may develop breast cancer or ovarian cancer. Only $5 \%$ of patients with breast cancer have a deleterious BRCA gene and these individuals have deficient DNA repair. Human cells have multiple mechanisms to repair damaged DNA. In BRCA-associated tumor cells, one of these pathways, a homologous recombination, is already inactivated because there is no working copy of either BRCA1 or BRCA2. The class of drugs called PARP (poly-ADP ribose polymerase) inhibitors inactivates a second pathway, a nonhomologous end-joining pathway, and this leaves the cell without two DNA repair pathways. This is often sufficient to leave the tumor cell unable to adequately repair new DNA errors and leads to tumor cell lethality. Olaparib is a PARP inhibitor that has been shown to improve progression-free survival in women with a deleterious mutation in BRCA and ovarian cancer [16]. It is currently under study in breast cancer in the OLYMPIA trial - women with a deleterious BRCA mutation and early-stage breast cancer who have completed chemotherapy are randomized to 5 years of olaparib versus placebo (NCT0203282; https//: clinicaltrials.gov).

Ongoing research is focused on understanding the mechanisms of drug response and drug resistance. The challenge of 2015 and beyond is 'precision medicine' - prevention and treatment strategies that take individual variability into account. The development of technologies to identify the precise aberrations of each individual's cancer so that appropriate therapies can be developed and administered is the challenge of the 21st century. The ability to address this goal has been dramatically improved by the recent development of large-scale biological databases (such as the human genome sequence), powerful methods for characterizing patients (such as proteomics, metabolomics, genomics, diverse cellular assays and even mobile health technology) and computational tools for analyzing large sets of data. In the State of the Union address on January 20, 2015, the US President Barrack Obama announced the launch of a new Precision Medicine Initiative: 'Tonight, I'm launching a new Precision Medicine Initiative to bring us closer to curing diseases like cancer and diabetes - and to give all of us access to the personalized information we need to keep ourselves and our families healthier.'

\section{Disclosure Statement}

The author declares that no conflicts of interest exist.

\section{References}

1 Ferlay J, Soerjomataram I, Ervik M, et al: GLOBOCAN 2012: Cancer Incidence and Mortality Worldwide: IARC CancerBase 11 (internet). Lyon, International Agency for Research on Cancer, 2013. http://globocan.iarc. fr (accessed October 28, 2015).

2 Favourable and unfavourable effects on longterm survival of radiotherapy for early breast cancer: an overview of the randomised trials. Early Breast Cancer Trialists' Collaborative Group. Lancet 2000;355:1757-1770.

3 Fisher B, Anderson S, Bryant J, et al: Twentyyear follow-up of a randomized trial comparing total mastectomy, lumpectomy, and lumpectomy plus irradiation for the treatment of invasive breast cancer. N Engl J Med 2002;347:1233-1241.

4 Sorlie T, Perou CM, Tibshirani R, et al: Gene expression patterns of breast carcinomas distinguish tumor subclasses with clinical implications. Proc Natl Acad Sci USA 2001;98: 10869-10874.

5 Fisher B, Jeong JH, Bryant J, et al: Treatment of lymph-node-negative, oestrogen-receptorpositive breast cancer: long-term findings from national surgical adjuvant breast and bowel project randomised clinical trials. Lancet $2004 ; 364: 858-868$.

6 BIG Collaborative Group; Mouridsen H, Giobbie-Hurder A, et al: Letrozole therapy alone or in sequence with tamoxifen in women with breast cancer. N Engl J Med 2009;361:766-776.

7 Coombes RC, Hall E, Gibson LJ, et al: A randomized trial of exemestane after two to three years of tamoxifen therapy in postmenopausal women with primary breast cancer. N Engl J Med 2004;350:1081-1092.

8 Goss PE, Ingle JN, Martino S, et al: A randomized trial of letrozole in postmenopausal women after five years of tamoxifen therapy for early-stage breast cancer. N Engl J Med 2003;349:1793-1802.

9 Pagani O, Regan MM, Walley BA, et al: Adjuvant exemestane with ovarian suppression in premenopausal breast cancer. N Engl J Med 2014;371:107-118.

10 Paik S, Shak S, Tang G, et al: A multigene assay to predict recurrence of tamoxifen-treated, node-negative breast cancer. N Engl J Med 2004;351:2817-2826.
11 Slamon D, Eiermann W, Robert N, et al: Adjuvant trastuzumab in HER2-positive breast cancer. N Engl J Med 2011;365:1273-1283.

12 Perez EA, Romond EH, Suman VJ, et al: Fouryear follow-up of trastuzumab plus adjuvant chemotherapy for operable human epidermal growth factor receptor 2-positive breast cancer: joint analysis of data from NCCTG N9831 and NSABP B-31. J Clin Oncol 2011;29:3366-3373.

13 Piccart-Gebhart MJ, Procter M, LeylandJones B, et al: Trastuzumab after adjuvant chemotherapy in HER2-positive breast cancer. N Engl J Med 2005;353:1659-1672.

14 Romond EH, Perez EA, Bryant J, et al: Trastuzumab plus adjuvant chemotherapy for operable HER2-positive breast cancer. N Engl J Med 2005;353:1673-1684.

15 Swain SM, Baselga J, Kim SB, et al: Pertuzumab, trastuzumab, and docetaxel in HER2positive metastatic breast cancer. N Engl J Med 2015;372:724-734.

16 Ledermann J, Harter P, Gourley C, et al: Olaparib maintenance therapy in platinumsensitive relapsed ovarian cancer. N Engl J Med 2012;366:1382-1392. 